\title{
A Software for Lab Access and Chemical Process Logging in Shared Facilities
}

\author{
Shuyou $\mathrm{Li}^{1}$ and Fernando Camino ${ }^{2}$ \\ ${ }^{1}$ FOM Networks, Inc. One Northfield Plaza, Suite 300, Northfield, IL 60093-1214, USA \\ ${ }^{2}$ Center for Functional Nanomaterials, Brookhaven National Laboratory, Upton, NY 11973, USA
}

Research safety management, including safety training, documentation, and daily logging, is always a concern especially in multidisciplinary shared facilities. Two years ago in the Microscopy and Microanalysis meeting (Indianapolis, 2013), we presented a software system we designed for Northwestern University for university-level safety management. With that safety management system, Northwestern University shared facilities can make sure every facility user, no matter which department the user comes from, must pass the required safety training before they can use the resources in the laboratory. This safety management system was at instrument level, i.e. an instrument manager may define which safety clearances are required for each individual resource in the laboratory [1].

In this paper we present another system we developed for the Center for Functional Nanomaterials, Brookhaven National Laboratory. As an add-on to the BNL's Facility Online Manager (FOM®) system [2], this software provides the lab access and daily chemical process logging in the cleanroom.

Figure 1 is a screenshot of the access control system. The access control station is usually located beside the main entrance of the laboratory, where every user must scan their ID cards to enter the lab. After scanning an ID card, the user's information is recorded in the main FOM® server and the laboratory can check this record or bill the user based on the time this user works inside the lab. Each user is also asked a random safety question every time they enter the lab. If a user fails any part of the safety questionnaire, the user will be banned from the lab and their name will be listed in the table of "Users who must see a staff member".

The logging of the chemical processes is done on a second computer inside the lab, where a user should record all chemical usage in the lab, as shown in Figure 2. Users can also check out safety documents such as the SOP or MSDS of any chemical at any time while working in the lab. As soon as a user starts a chemical process, the record is shown in the first lab, where the lab manager or another user may check whenever needed. After doing a chemical process, the user must click the End Process button to finish using a chemical. Otherwise their name will be listed in the "Users who forgot to END chemical process".

A lab manager can also see the list of current users in the lab, records of chemical usage. Lab managers can also manage users and resources and edit the tip of the week, recent changes, and safety questionnaire items on their office computers using the main FOM® system.

\section{References:}

[1] S. Li, S.V. Mallipeddi, S. Karlman, T. Moskal and V.P. Dravid (2013). Safety Management in Multidisciplinary Shared Facilities. Microscopy and Microanalysis, 19 (Suppl. 2), pp 1396-1397.

[2] See more details about the Facility Online Manager® software at http://www.fomnetworks.com/. 


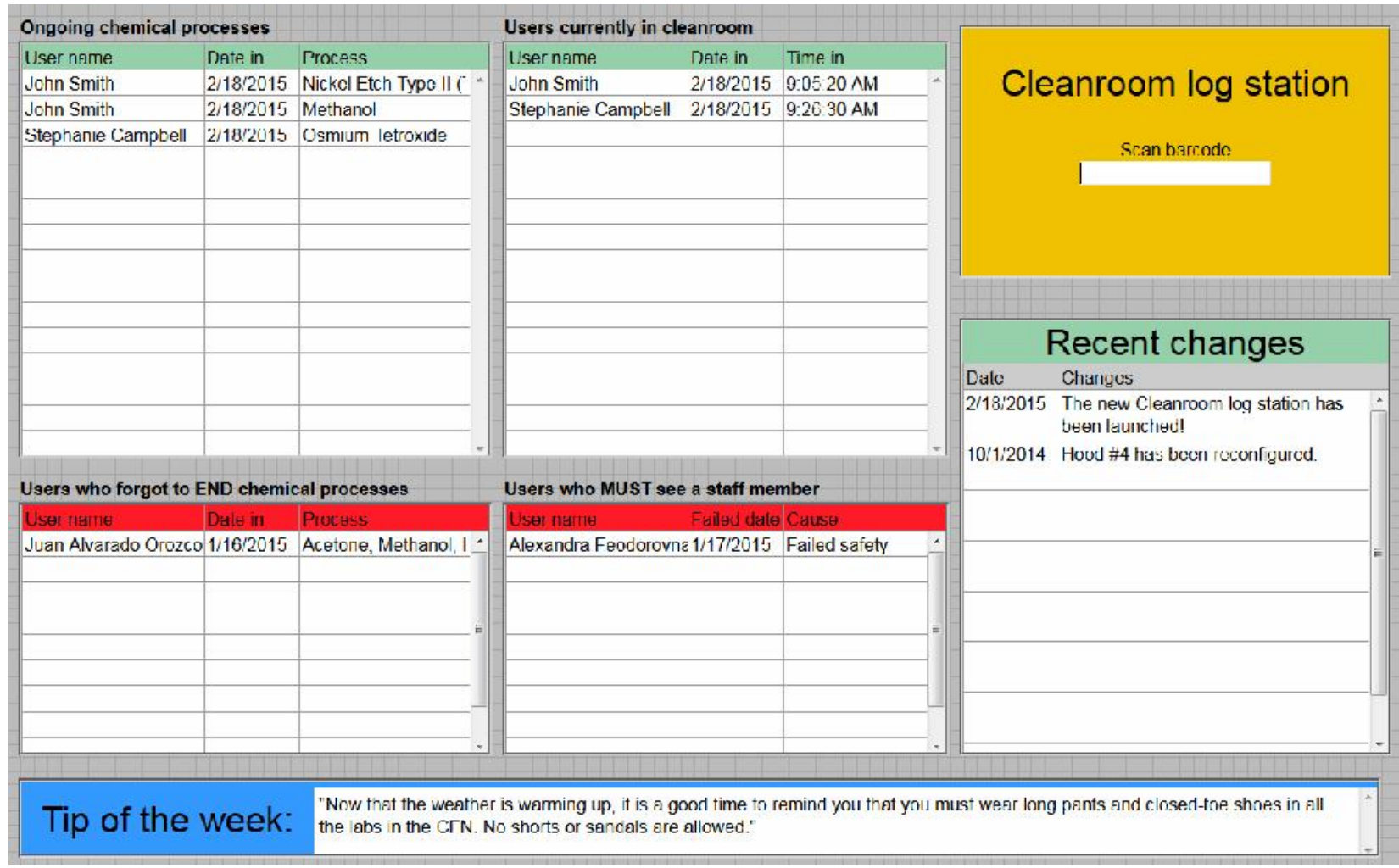

Figure 1. A screenshot showing the access control and chemical processes log station in a cleanroom.

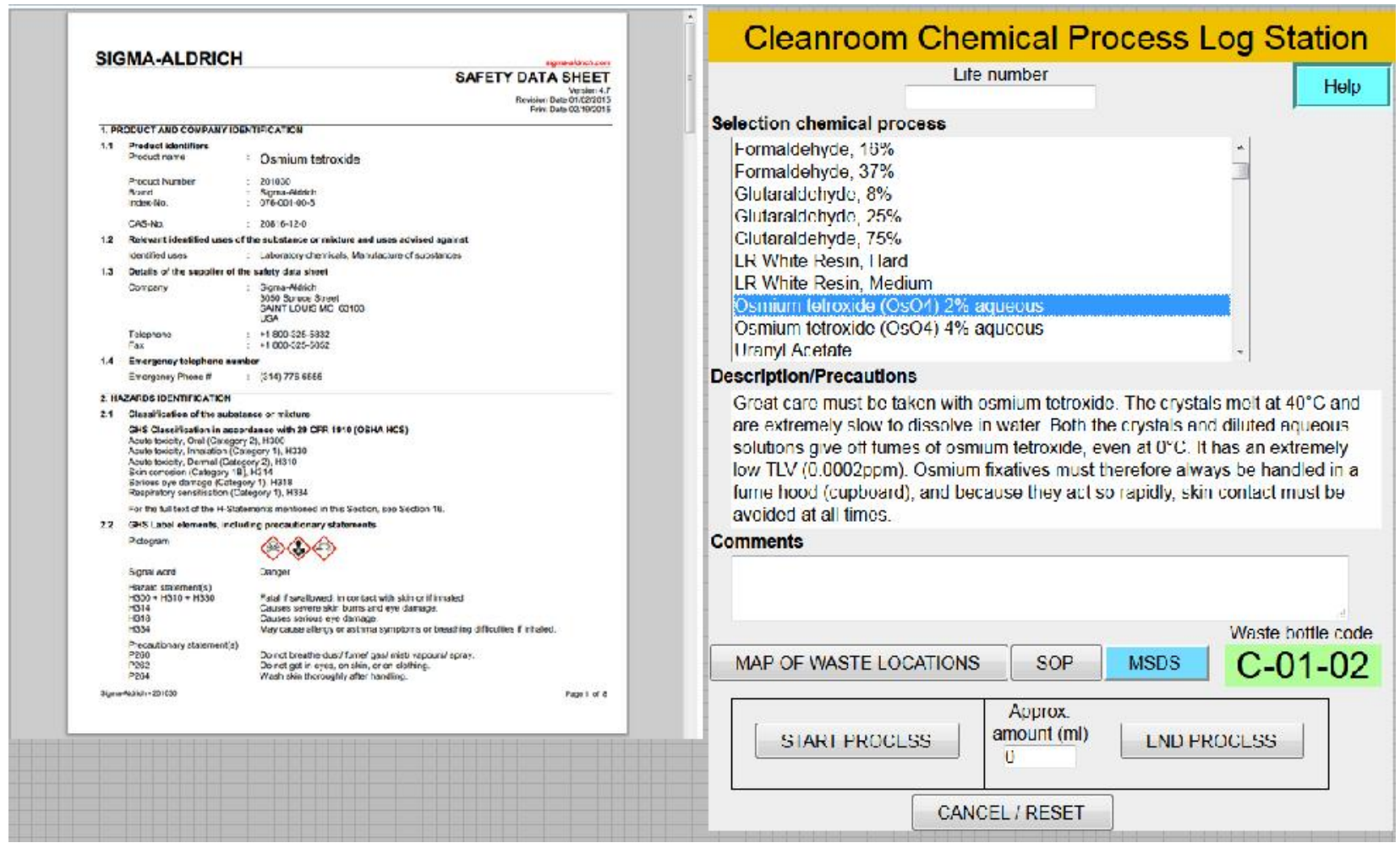

Figure 2. A screenshot showing the chemical logging system in a cleanroom. 